\title{
Cuidados paliativos $y$ eutanasia: especial referencia a la legislación belga
}

ANA MARIA MARCOS DEL CANO

Profesora Titular de Universidad de Filosofla del Derecho

de la Facultad de Derecho, UNED

No cabe duda de que uno de los temas más debatidos en los últimos años es la legitimidad de la reivindicación hecha por parte de un sector de nuestra sociedad del derecho a morir con dignidad. Derecho que, en una primera aproximación, nos resulta extraño, ¿derecho a morir? Normalmente, cuando se reclama un derecho es a algo bueno, beneficioso y, en principio, la muerte se nos presenta cuando menos como un mal, aunque por desconocida, sería más ajustado decir que es algo incierto. Como afirma G. Bueno: "¿Cómo pueden mantenerse reunidos, o dentro de qué condiciones, los conceptos de "bueno" y "muerte"? Pues, ¿̇acaso la muerte puede llamarse buena en alguna circunstancia? ¿no es siempre mala? Más aún: ¿̇no es la muerte, con su siniestra guadaña, el símbolo mismo del mal? Buena muerte, eutanasia, ¿no es algo así como el "círculo cuadrado"? Además, si afinamos más en la expresión, no se puede afirmar que tengamos derecho. La muerte, mal que nos pese, es una realidad a la que irremediablemente vamos a llegar y de la que una vez "arrojados a la vida", como decía Ortega, ya no nos podemos librar. El derecho a morir implica como tal derecho una actitud valorativa, rei- 
vindicativa de fondo, que reclama la protección y la garantía de un "buen morir", dada la situación extrema en la que se encuentran algunos enfermos terminales.

Pero es que en este caso aparece junto al derecho a morir un adjetivo que, si se me permite la expresión, nos viene a complicar las cosas. En principio, todos deseamos una muerte digna. La cuestión radica en qué situaciones concretas se puede vulnerar esa dignidad de la muerte y ahí surge la dificultad, precisamente, porque el paso del plano descriptivo al normativo es muy habitual en estos casos. ¿Qué quiero decir? Pues, sencillamente, que muchas de las definiciones de ese derecho a morir con dignidad son parciales o subjetivas, con el fin de derivar de ellas su posición normativa. Así, para unos, derecho a morir con dignidad significa poder decidir el momento de la muerte; para otros, será el morir de un modo natural, sin necesidad de intervenir ${ }^{2}$.

Con todo esto quiero poner de manifiesto que la expresión derecho a morir con dignidad o su sinónimo, la eutanasia, no es pacífica, sino más bien todo lo contrario: muy controvertida y susceptible de diferentes y antagónicos significados ${ }^{3}$.

No obstante, lo que sí parece que ha logrado un cierto consenso es que la dignidad en el morir implica el no sometimiento al encarnizamiento terapéutico. La dificultad surge cuando a esta situación se llega por diferentes vías: unos, abogan por adelantar el momento de la muerte -eutanasia-; otros, en cambio, son partidarios de acudir a cuidados paliativos -ortoeutanasia, eutanasia lenitiva ${ }^{4}$ o simplemente dejar morir.

Estas dificultades que presenta el lenguaje con el que "nombramos" estas situaciones traen el fondo de la cuestión: la complejidad del tema que tenemos entre manos y la ambigüedad con que los términos se emplean. Como a nadie se le escapa a esto se añade el hecho de que hay varias disciplinas o ramas implicadas en su tratamiento: medicina, ética, derecho, psicología... Y, por otro lado, toca los valores más básicos de la existencia humana, a saber, la vida, la libertad, la dignidad. Además las connotaciones ideológicas, emocionales y sentimentales tergiversan en 
muchas ocasiones la objetividad en las distintas propuestas del debate. Debate que no puede ser sólo teórico, sino que necesita bajar a las circunstancias del caso concreto para dar una solución adecuada.

Esta problemática se agudiza cuando nos adentramos en el campo jurídico. Aparte de las connotaciones éticas y sociales que están en juego, nos hallamos con gran dificultad para lograr una regla general aplicable a todas las hipótesis imaginables. Toda la legitimación de la eutanasia se hace girar sobre dos pivotes: el sufrimiento (la baja calidad de vida) y el consentimiento del paciente. Por un lado, el sufrimiento tanto físico como psíquico y, sobre todo, la vivencia de ese dolor hace que no existan dos casos iguales y que sea muy difícil el medir la "insoportabilidad" de ese sufrimiento. Por el otro, dadas las circunstancias en las que se halla el enfermo es muy posible que se encuentre con una autonomía reducida para expresar un consentimiento válido jurídicamente. Además de que, en muchos casos, se nos advierte desde la psicología que esos "deseos" o "peticiones" pueden ser solamente un reclamo de ayuda, de atención, de cariño. No hay que olvidar tampoco, como ya he puesto de manifiesto en otros ámbitos, que hay un tercero que materializa la acción y cuya posición no es meramente la de un "agente pasivo", sino que debe interpretar esos deseos del enfermo. Además, la investigación en Medicina está en constante avance, lo cual crea ya una gran incertidumbre en relación con los pronósticos de algunas enfermedades ${ }^{5}$.

Aún con toda esta problemática algunos países han adoptado ya legislaciones concretas. El último en hacerlo -el primero fue Holanda- ha sido Bélgica. Me interesa resaltar la legislación belga porque en ella como vamos a ver a continuación se recoge la realidad de la ayuda a morir desde la atención integral al paciente terminal, ofreciéndole por un lado, la posibilidad de los cuidados paliativos y sólo después de que hayan sido aplicados, se podrá solicitar la eutanasia. Veamoslo más detenidamente. 
El tema de la eutanasia presenta un gran calado en las sociedades modernas, sobre todo, por el creciente envejecimiento de la población, por un lado, y el aumento de la duración de la vida de enfermos con una afección incurable.

El fenómeno de la eutanasia en Bélgica no es nada extraño. Al menos, así se desprende de los datos que ofrece una reciente encuesta elaborada por investigadores de la Universidad Vrije de Bruselas y de la Universidad Rijks de Gante que revelan que el $40 \%$ de los fallecimientos son intervenidos prematuramente a continuación de una decisión médica. En el $17 \%$ de los casos, se ha interrumpido el tratamiento, en un $18 \%$ se han administrado a los pacientes medicamentos analgésicos que podían tener el efecto de acortar la vida y, en un $4 \%$ de los casos los enfermos han recibido medicamentos letales. En este último supuesto, el problema es que 3 de los 4 pacientes a los que se suministró la dosis letal, no lo habían pedido. Ante esta situación, surgió la necesidad de abordar la regulación jurídica de las actuaciones al final de la vida y, en consecuencia, del "espinoso" tema de la eutanasia.

La proposición de ley sobre la eutanasia en Bélgica se presentó en 1999 por parte de seis senadores y suscitó violentas reacciones, sobre todo por parte de los partidos políticos más conservadores. Después de un intenso debate en comisiones de Justicia y de Asuntos Sociales que examinaron la proposición de ley, se decidió organizar audiciones. Unas cuarenta personas fueron escuchadas, además de ser retransmitidas por televisión. Las exposiciones y testimonios de especialistas en ética, de médicos, psicólogos, cuidadores, pacientes y juristas fueron chocantes. Las certidumbres se tambaleaban. Sin embargo, los senadores consideraban que una legislación sobre cuestiones éticas tan importantes debía lograr un amplio consenso y que, por lo tanto, había que reelaborar la proposición de ley de los senadores. Tras múltiples e intensos debates, se aprobó la ley el 28 de mayo de 2002 y fue publicada el 22 de junio de 2002 en el Moniteur Belge ${ }^{6}$. 
Bélgica, siguiendo en gran medida los pasos de su vecina Holanda, ha apostado por una legislación sobre la eutanasia novedosa, y novedosa por lo siguiente: ha elaborado a la par de la ley sobre la eutanasia, una legislación sobre los cuidados paliativos ${ }^{7}$, de tal modo que para poder solicitar la eutanasia, previamente el enfermo terminal ha tenido que acudir a los cuidados paliativos para evitar en la medida de la posible los dolores que le produce su enfermedad. Y sólo en el caso de que estos no dieran los resultados esperados, podría pedir la eutanasia. Me parece una medida adecuada en tanto que relega la práctica de la eutanasia como último recurso.

Veamos cómo se ha desarrollado el debate en Bélgica sobre esta cuestión.

\subsection{CONCEPTO DE EUTANASIA}

El Comité de Bioética de Bélgica definió la eutanasia como el acto practicado por un tercero, que pone fin intencionalmente a la vida de una persona bajo su demanda. Aparentemente, esta definición no se circunscribiría a los casos de eutanasia pues en ella nada se dice ni de enfermedad terminal, ni de sufrimientos insoportables, bien podría ser la definición de la ayuda al suicidio.

En la ley que se acaba de aprobar en mayo de 2002 y publicada en junio recoge también la misma definición, en su artículo 2 establece que "para la aplicación de la presente ley, se entenderá por eutanasia el acto, practicado por un tercero, que pone fin intencionalmente a la vida de una persona bajo su demanda". No obstante, en los artículos siguientes concreta las condiciones y el procedimiento en los que ya figuran las circunstancias de la eutanasia.

\section{a) Sujeto pasivo}

Concretar quien sea o pueda ser el sujeto pasivo en esta relación jurídica es de una importancia central en el desarrollo de la problemática jurídica de la eutanasia. ¿Por qué? El hecho de determinar el grupo de individuos que pueden solicitar la eutanasia supone acotar y restringir 
su aplicación a unos supuestos muy concretos. En relación con este punto se suscitan tres cuestiones, a saber, la determinación de la muerte; la irreversibilidad y terminalidad de la enfermedad y, por último, la capacidad del enfermo.

En cuanto al primer punto, nos situamos ante una persona viva que presenta una breve expectativa de vida, alrededor de 6 meses. Hago hincapié en la palabra "viva" porque en muchas ocasiones las fronteras entre la vida y la muerte en esta última fase se presentan muy difusas. En este contexto y con el fin de diferenciarlo de otros supuestos posibles es preciso tener presente cuando estamos en presencia de la muerte. Desde el punto de vista jurídico, es de gran transcendencia establecer con exactitud cuándo se produce este momento. De su fijación dependerá que un supuesto atentado a la vida humana constituya o no delito ${ }^{8}$. Al Derecho lo que le interesa es averiguar si existe todavía un ser humano con vida, vida que debe proteger, y para tal fin serían suficientes criterios médicos aceptados por la generalidad.

$\mathrm{Al}$ consistir la muerte en un proceso irreversible habrá que seleccionar un punto del conjunto de dicho proceso en el que se produzca la lesión irreversible e irrecuperable de alguna función vital del cuerpo humano, aunque otras partes concretas mantengan todavía la existencia biológica9 . Los avances de la ciencia médica han puesto de manifiesto que un deterioro sustancial del cerebro es completamente irrecuperable, esto es, que cuando se produce la muerte cerebral puede entenderse muerta a una persona, pues las funciones del cerebro son rectoras de otras del organismo sin las cuales éste no puede seguir actuando autónomamente, incluso aunque éstas puedan ser mantenidas de forma artificial $o$ asistida ${ }^{10}$.

En cuanto al segundo punto, es preciso, a mi entender, circunscribir los supuestos de eutanasia a los enfermos terminales, pues ya en el origen de la palabra "eutanasia", encontramos las ideas de ausencia de dolor en el morir cuando está próxima la muerte. Por otra parte, la situación en la que se están los enfermos terminales difiere de las que se 
enmarcan en otros supuestos (como los tetrapléjicos, los recién nacidos...). Por ejemplo, la expectativa de vida, la capacidad del individuo, la calidad de vida, el estado de irreversibilidad... ${ }^{11}$ Entiendo que enfermo terminal es aquella persona que, como consecuencia de una lesión o enfermedad, padece, según los actuales conocimientos médicos, una afección incurable y se encuentra en la fase final de su vida sin ninguna esperanza de recuperación (normalmente, el plazo se suele establecer en unos seis meses). En general, se encuentran en esta situación los enfermos de cáncer avanzado, en los que la enfermedad ni puede ser controlada ni existen planes para continuar con los tratamientos curativos.

Por lo que se refiere a la tercera cuestión, se suscita el problema del posible deterioro de la capacidad de los enfermos terminales para prestar su consentimiento. ¿Puede hablarse de verdadera capacidad, teniendo en cuenta las circunstancias en las que la enfermedad sitúa a estas personas? ¿La enfermedad terminal y la plena capacidad de obrar son compatibles? Es lógico que al menos nos cuestionemos esto pues hay momentos en los que el paciente atraviesa por una profunda depresión o su consciencia se halla disminuida como consecuencia de los efectos de los fármacos administrados o del propio estado derivado del proceso de su enfermedad. En principio y como premisa para este debate creo que hay que partir del hecho de que es demasiado simplista afirmar por regla general que el enfermo terminal es incapaz para prestar su consentimiento. Habrá que estar al caso concreto y analizarlo con detenimiento.

¿Cómo se recogen estos puntos en la legislación belga?

En la legislación belga el sujeto pasivo de la eutanasia sería aquella persona mayor de edad o menor emancipado, capaz y consciente en el momento de presentar su petición y además que se encuentre en una situación médica sin salida y con un sufrimiento físico o psíquico constante e insoportable que no puede ser calmado. (art. 3.1) De esta redacción no se desprende claramente cuál tiene que ser la situación objetiva 
del paciente, pudiendo ser la de enfermedad terminal o no, puesto que simplemente hace referencia a una situación médica sin salida, que bien puede ser una enfermedad irreversible sin que tenga que ser terminal necesariamente. Dejaría, pues, abierta la aplicación de la eutanasia a enfermos terminales, irreversibles pero no terminales. Es asimismo indispensable la capacidad plena para solicitarla, aunque como veremos a continuación, en el capítulo tercero, se prevé que dicha voluntad se haya expresado anticipadamente.

b) Sujeto activo

El debate que se suscita en este punto es si debe ser el médico o alguien cualificado quien realice esta acción. La primera cuestión que surge es ipor qué debe ser el médico? Las razones que se han esgrimido a favor de que una ley sobre la eutanasia prevea que sea el médico el que la practique son las siguientes: a) actualmente, el médico se encontrará casi siempre en el centro de la escena dado que la muerte actualmente acaece en los hospitales; b) con el fin de dotar de una mayor seguridad y control a la práctica de la eutanasia; c) además, es el médico quien mejor conoce los fármacos que pueden hacer el tránsito más dulce (etimológicamente, eutanasia, deriva del griego $-\mathrm{eu}=$ bueno y Thanatós $=$ muerte -$)^{12}$.

La segunda cuestión, como no podía ser de otra manera, es la de la objeción de conciencia del personal sanitario, es decir, el incumplimiento de un deber jurídico motivado por la existencia de un dictamen de conciencia, que impide observar el comportamiento que la norma prescribe por motivos morales individuales. Si se llegara a establecer por ley que el médico debiera aplicar la eutanasia a los pacientes que cumplan ciertos requisitos previstos legalmente, será preceptivo incorporar a tal normativa una cláusula de objeción de conciencia para los que no puedan practicar la eutanasia debido a sus convicciones morales. De otro modo, se estaría incurriendo en discriminación ${ }^{13}$.

En la legislación belga aparece en todo momento que será el médico quien practique la eutanasia, no cabe otra posibilidad. Es más, su artículo 3.1 comienza con una exención de responsabilidad penal del mé- 
dico que la realice ${ }^{14}$. Además, en el art. 14 recoge la objeción de conciencia del personal médico, estableciendo que quien rechace una petición de eutanasia deberá informar al paciente o a la persona de confianza y dar traslado del dossier médico al médico designado por el paciente o dicha persona.

c) Conducta

Por lo que respecta a la acción queda claro que la legislación belga se refiere únicamente a aquellas conductas activas. De hecho las omisiones en estos casos constituyen tratamientos incluidos dentro de la lex artis del médico ${ }^{15}$. En su artículo 2 dice textualmente "eutanasia es el acto".

d) Procedimiento

El procedimiento comienza con la petición que el paciente deberá realizar que tendrá que ser "de manera voluntaria, reflexionada y reiterada" (art. 3.1) y presentarla por escrito (art. 3.4).

El médico deberá informar al paciente de su estado de salud y de su esperanza de vida, así como de las posibilidades terapéuticas y las que ofrecen los cuidados paliativos. Ambos deben llegar a la convicción de que no hay otra solución en su situación y que la decisión del paciente es totalmente voluntaria. Deberá consultar a otros dos facultativos antes de practicarla y tiene que dejar pasar al menos un mes desde que el paciente hizo la petición por escrito antes de llevarla a cabo.

También se prevé la posibilidad de expresar la voluntad de un modo anticipado (se dedica el capítulo III de la ley). En aquellos caos en los que el paciente es inconsciente puede designar a una o varias personas de confianza, mayores de edad, clasificadas por orden de preferencia, que pongan al médico al corriente de la voluntad del paciente. La declaración puede hacerse en cualquier momento, por escrito y en presencia de dos testigos mayores de edad. Esta declaración puede revocarse en cualquier momento ${ }^{16}$

Una vez realizada, se considera que el paciente ha fallecido de muerte natural. Cuatro días después, el médico deberá presentar un documento para su registro a la comisión Federal de control y de Evaluación 
de la aplicación de la ley de la eutanasia, compuesta por 16 miembros, que se encargará de verificar que ha sido efectuada según lo previsto en la ley ${ }^{17}$. En el caso que dos tercios de los miembros de dicha Comisión estimen que el doctor no se ha ceñido a las condiciones fijadas por la ley, dicho órgano enviará el caso al fiscal del tribunal donde se haya producido el fallecimiento para que este emprenda medidas judiciales si lo considera oportuno.

\subsection{REFERENCIA A LOS CUIDADOS PALIATIVOS}

El procedimiento es similar al de su país vecino, Holanda. Las diferencias cualitativas que señalaría serían las siguientes. En primer lugar, se habla en todo caso de eutanasia, no de ayuda al suicidio ni de terminación de la vida a petición propia; y, en segundo lugar, la ley belga no autoriza la práctica de la eutanasia a los menores no emancipados. La diferencia más importante, a mi entender, sin embargo, es la necesidad de acudir previamente a los cuidados paliativos para tratar de mitigar el sufrimiento. En el art. 3 establece que el médico debe comunicar al paciente las posibilidades que ofrecen los cuidados paliativos y juntos deben llegar a la convicción de que no existe ninguna solución razonable en su situación.

De hecho y como apunté al inicio, la novedad más sobresaliente, a mi entender, es que junto a la ley de despenalización de la eutanasia se ha aprobado la ley de cuidados paliativos.

En Bélgica el desarrollo de los cuidados paliativos se debió principalmente a las iniciativas privadas de asociaciones tales como "Continuing Care" y el Centro para la Asistencia de Enfermos moribundos ${ }^{18}$. La primera unidad de cuidados paliativos se crea en 1990. Además la Federación belga de asociaciones de cuidados paliativos llevó a cabo una acción dinámica para promover los cuidados en el domicilio igual que en el hospital y ofrecer a los médicos una formación específica.

En 1999, el 20 de diciembre se presentó la proposición de ley. En sus fundamentos se afirma que los cuidados paliativos representan para los 
pacientes y la medicina un progreso importante que integra las dimensiones físicas, psicológicas y social de las personas que padecen una enfermedad incurable. La presente Ley publicada el 26 de octubre de 2002 en el Moniteur Belge se articula en tres partes:

1.- Trata de asegurar el derecho de un paciente a la posibilidad de beneficiarse de los cuidados paliativos.

2.- Incluye la indicación al Gobierno de los medios que es necesario promover para alcanzar tal objetivo.

3.- La afirmación del derecho del paciente de recibir la información exacta y completa en cuanto al diagnóstico del médico y a las previsiones de la evolución de la enfermedad.

Es una lástima que en la reciente Ley básica sobre los derechos de los pacientes española del 14 de noviembre de $2002^{19}$ no se haya abordado esta cuestión y simplemente figure en su art. 21 cuando habla del alta del paciente lo relativo a los cuidados de carácter paliativo. En concreto afirma que "en caso de no aceptar el tratamiento prescrito, se propondrá al paciente o usuario la firma del alta voluntaria. Si no la firmara, la dirección del centro sanitario, a propuesta del médico responsable, podrá disponer el alta forzosa en las condiciones reguladas por la Ley. El hecho de no aceptar el tratamiento prescrito no dará lugar al alta forzosa cuando existan tratamientos alternativos, aunque tengan carácter paliativo, siempre que los preste el centro sanitario y el paciente acepte recibirlos. Estas circunstancias quedarán debidamente documentadas."

En este sentido, creo que en última instancia lo que debe hacer el debate sobre la eutanasia es suscitar una mayor sensibilidad hacia los derechos de los enfermos y los moribundos, hacia su dignidad y tratar sobre todo de ayudarles en el morir. Ineludiblemente creo que como Bélgica, como también lo ha hecho Francia ${ }^{20}$, el abordar la legislación sobre la eutanasia tiene que pasar por una legislación sobre los cuidados paliativos, por la promoción de los medios y recursos necesarios para su cobertura social. 
1 En G. BUENO, El sentido de la vida. Seis lecturas de filosofia moral, Oviedo, Pentalfa, 1996, p. 201.

2 Véase, MARCOS DEL CANO, A.M., La eutanasia. Estudio filosófico-juridico, Madrid, Marcial Pons-UNED, 1999, pp. 32-42.

3 Véase MARCOS DEL CANO, A.M., "Dignidad humana en el final de la vida y cuidados paliativos", en MART'ÍNEZ MORÁN, N. (coord.), Biotecnologia, Derecho y Dignidad humana, Granada, Comares, 2003, pp. 237257.

4 Algunos autores hablan de ettanasia lenitiva o indirecta para referirse a las situaciones en las que se administran medicamentos o terapias al enfermo, con el fin primordial de mitigar sus sufrimientos, aun sabiendo que, como efecto secundario, es ineludible el acortamiento de su vida. $A$ mi entender, es preferible dejar el término eutanasia solamente para aquellos casos de terminación activa de la vida de un enfermo terminal, bajo su petición.

5 Véase mi trabajo La eutanasia...,1999.

6 De acuerdo con su última disposición particular la ley entrará en vigor como muy tarde a los tres meses de su publicación en el Moniteur Belge. Ver, http://www.moniteur.he

7 La proposición de ley sobre la eutanasia se presentó en 1999 y el mismo año el 20 de diciembre se presentó el proyecto de ley sobure cuidados paliativos. Ver ht1p://www.senal,be

8 Evidentemente el determinar tal momento no es tarea ya de los juristas, sino que ha des ser fruto de las aportaciones de los investigadores de las Ciencias Biomédicas; es una información que sólo los expertos están en condiciones de suministrar con sus métodos científicos propios. Sin embargo, es cierto que el Derecho se desenvuelve propiamente en lo axiológico, en el mundo de los valores, y a él compete proceder a las valoraciones oportunas de esa información de las Ciencias Biomédicas, operando también con los métodos que le son propios y los propósitos normativos que le son propios y los propósitss normativos que le caracterizan, y es en esta tarea en la que el jurista -en cuanto experto- puede contribuir con su específica preparación. Deberá dehatirse para decidir hasta qué momento corresponde la protección jurídica de la vida humana. En último extremo es un problema normativo, pero que debe partir en todo caso de la realidad ontológica que se desea acotar. Sobre esto, ver el exhaustivo estudio de ROMEO CASABONA, C.M., El Derecho y la Bioética ante los límites de la vida humana, Madrid, Centro de Estudios De Ramón Areces, 1995, pp. 161-183.

9 El concepto de muerte legal se recoge en nuestra legislación en el Real Decreto 2070/99, de 30 de diciembre (BOE, 4-1-2000) donde se regulan las actividades de obteneión y utilización clínica de órganos humanos, incluida la donación, extracción, preparación. transporte, distribución y las de transplante y su seguimiento.

10 La muerte cerebral consiste básicamente en que cesan las funciones corticales (conciencia) y las del encéfalo (funciones vegetativas, reflejos primarios -ej. Respiración espontánea, tos, pupilares...). Persiste el ritmo cardiaco autónomo. En cambio, el estado vegetativo persistente (EVP) consiste en la muerte cortical, es decir, está inconsciente pero despierto (con los ojos abiertos, mantiene el sueño-vigilia y los reflejos primarios.

11 Para profundizar más sobre estas cuestiones, MARCOS DEL CANO, La eutanasia..., 1999 , pp. 196 y 85.

12 En este sentido, el art. 143.4 del Código Penal español recoge la tipificación de la eutanasia y, sin embargo, no hace ninguna mención al médico: "El que causare o cooperare activamente con actos necesarios $y$ directos a la muerte de otru, por la petición expresa, serie e 
inequivoca de éste, en el caso de que la victima sufriera una enfermedad grave que conduciria necesariamente a su muerte, o que produjera graves padecimientos permanentes y dificiles de soportar, será castigado con la pena inferior en uno o dos grados a las señaladas en los números 2 y 3 de este articulo". Sobre la génesis y la problemática de este artículo en los distintos proyectos, vid. MARCOS DEL. CANO, A.M., "Eutanasia: relevancia jurídicopenal del consentimiento", Cuadernos de Bioética, nº, 4", 1993, pp. 53-62. No ocurre lo mismo, sin embargo, en Holanda, país en el que se recoge la despenalización de la eutanasia, pero debe ser el médico quien tras un minucioso proceso la lleve a cabo. Para obtener más información sobre la regulación jurídica de la eutanasia en Holanda, consultar MARCOS DEL CANO, A.M., "La eutanasia en el ordenamiento jurídico holandés", Cuadernos de Bioética, vol. VII, $\mathbf{n}^{\circ} 27,3^{2}, 1996$, pp. 319328; ampliado y revisado con las últimas modificacioncs jurisprudenciales y legislativas en MARCOS DEL CANO, A.M., "Legislación eutanásica y realidad social: la experiencia de Holanda", en ANSUÁTEGLI ROIG, FJ., Problemas de la entanasia, Madrid, DykinsonUniversidad Carlos III, 1999, pp. 72-85. En este sentido, en el Estado norteamericano de Oregón en 1994 se votó mayoritariamente a favor de una propuesta para legalizar el suicidio asistido por un médico. La ley ha tenido una vigencia de unos seis meses debido a los continuos recursos a los que está sometida. No obstante, lo único que prevé esta ley es la facultad de solicitar al médico la prescripción de una "sustancia letal" que, posteriormente, se suministrará el enfermo. Por otra parte y cambiando de continente, el 25 de mayo de 1995, el Parlamento del Territorio del Norte, en Australia, dio un paso singular al legalizar directamente la eutanasia voluntaria a través de la promulgación de la "Ley de los Derechos de los Enfermos en fase terminal", en la que se establece que un mayor de edad puede solicitar a su médico que ponga fin a su vida en caso de que padezca una enfermedad terminal. Sin embargo, tampoco ha entrado en vigor por las numerosas enmiendas a las que ha sido sometida. Sobre estas dos últimas, EIJK, W'J., "Eutanasia: contra el quinto mandamiento", Cuadernos de Bioética, 1996, 3², pp. 311-318.

13 Art. 14 de la CE: "...sin que pueda prevalecer discriminación alguna por razón de... religión, opinión o cualquier otra condición o circunstancia personal o social".

14 "El médico que practica una eutanasia no comete infracción si se asegura de que ..."

15 La problemática sohre la eutanasia activa y pasiva ha sido ampliamente debatida en los más diversos foros. Ver F.J. ANSUÁTEGUI ROIG, "Eutanasia: dilemas relevantes", en l:.J. ANSUÁTEGII ROIG (coordinador), Problemas de la eutanasia, DykinsonUniversidad Carlos III, Madrid, 1999, pp. 88122, en donde se recoge esta cruda polémica.

16 En este sentido, la reciente legislación española, la IEY 41/2002, de 14 de noviembre, básica reguladora de la autonomía del paciente y de derechos y obligaciones en materia de información ydocumentación clínica recoge también esta posibilidad (Instrucciones previas) para los tratamientos al final de: la vida, en su art. 11.1: "Por el documento de instrucciones previas, una persona mayor de edad, capaz y libre, manifiesta anticipadamente su voluntad, con objeto de que ésta secumpla en el momento en que llegue a situaciones en cuyas circunstancias no sea capaz de expresarlos personalmente, sobre los cuidados y el tratamiento de su salud o, una vez llegado el fallecimiento, sobre el destino de su cuerpo o de los órganos del mismo. El otorgante del documento puede designar, además, un representante para que, llegado el caso, sirva como interlocutor suyo con el médico o el equipo sanitario para 
procurar el cumplimiento de las instrucciones previas". Previamente, las Comunidades de Cataluña, Galicia y Extremadura ya habían adoptado sendas regulaciones. Ver MARCOS DEL CANO, A.M., "La toma de decisiones al final de la vida: el testamento vital y las indicaciones previas", Moralia, vol. XXIV2001-4, pp. 491-518.

17 En el art. 6 de la ley se recoge la composición de la comisión, su organización interna. El art. 7 establece el documento de registro que debe ser completado por el médico cada vez que practique una eutanasia. En él se recogen, entre otros, todos los datos particulares del paciente, de su enfermedad, la naturaleza de su sufrimiento, las razones por las que ese sufrimiento se calificó como no aplacable, las personas consultadas por el médico, así como los datos resultantes de tales consultas, si existe declaración anticipada y si designa alguna persona de confianza, señalar su nombre y apellidos, etc.

18 Ver CH. BYCK, "Soins palliatifs et politiques de santé Europe", Journal International de Bioéthique, 2002, vol. 13, n.1, p. 47.

19 Ley 41/2002, de 14 de noviembre, básica reguladora de la autonomía del paciente y de derechos $y$ obligaciones en materia de información y documentación clínica, en $B O E$, núm. 274, 15 noviembre 2002.

20 Ley sobre cuidados paliativos, del 9 de junio de 1999. También en este sentido el Consejo de Europa ha mostrado una especial sensibilidad en torno a los derechos de los moribundos con su Recomendación del Consejo de Europa relativa a la protección de los derechos del hombre y de la dignidad de los enfermos terminales y los moribundos, núm. 1418, 25 de junio de 1999. 